\title{
CYSTIC SCHWANNOMA OF THE ORBIT
}

\author{
DENNIS S. C. LAM ${ }^{1}$, JOAN S. K. NG ${ }^{1}$, KA F. TO ${ }^{2}$, VICTOR ABDULAH ${ }^{3}$, C. T. LIEW ${ }^{2}$ and \\ MARK O. M. TSO ${ }^{1}$ \\ Shatin, Hong Kong
}

\begin{abstract}
SUMMARY
Cystic schwannoma of the orbit is unreported in the world literature. A 54-year-old Caucasian man presented to our clinic with a 5 year history of progressive right-sided proptosis and diplopia. A large paramedian cystic mass displacing the right globe downwards and laterally was demonstrated. From the clinical and radiological features, the presumptive diagnosis of right frontal sinus mucocoele was made. However, the excisional biopsy of the lesion revealed the rare diagnosis of cystic schwannoma, arising from a branch of the first division of the trigeminal nerve. Postoperatively, the patient had a smooth recovery with visual acuity of 20/20 in each eye; full binocular single vision was also re-established. The differential diagnoses of cystic orbital mass, and the radiological and histological findings of the lesion, are described and discussed.
\end{abstract}

Schwannomas of the orbit are rare, accounting for $1 \%$ of all orbital tumours. ${ }^{1}$ This benign Schwann cell tumour of peripheral nerves usually presents as a slow-growing tumour in adulthood. The age of presentation ranges from 20 to 70 years. There is no predilection for sex or laterality. Most cases are unilateral and solitary. Slowly progressive painless ocular proptosis is the usual complaint. The direction of the proptosis may vary, but in general there is downward displacement of the globe, as most schwannomas arise from branches of either the supraorbital or supratrochlear nerve. Lesions located near the orbital apex may produce or simulate a retrobulbar neuritis. ${ }^{2}$ Neglected tumours may attain a phenomenal size, displacing the eye and producing diplopia.

From: ${ }^{1}$ Department of Ophthalmology and Visual Sciences, ${ }^{2}$ Department of Anatomical \& Cellular Pathology, and ${ }^{3}$ Department of Surgery (Otorhinolaryngology team), The Chinese University of Hong Kong, Prince of Wales Hospital, Shatin, N.T., Hong Kong.

Correspondence to: Dr Dennis S. C. Lam, Department of Ophthalmology and Visual Sciences, The Chinese University of Hong Kong, Prince of Wales Hospital, Shatin, N.T., Hong Kong. Tel: (852) 2632 2881. Fax: (852) 26483589.
Although most orbital schwannomas are not associated with neurofibromatosis, patients with these lesions may sometimes have pigmented cutaneous macules (cafe-au-lait spots). ${ }^{3}$ Malignant transformation of benign orbital schwannomas is extremely rare; nevertheless, it has been known to occur in patients with neurofibromatosis. ${ }^{4}$

The cystic form of schwannoma is very rare, but has been reported to occur in the brainstem, ${ }^{5}$ intracranial cranial nerves ${ }^{6,7}$ and adrenal region. ${ }^{8}$ We report here a patient with pure cystic form of schwannoma arising from the orbit.

\section{CASE REPORT}

A 54-year-old Caucasian man presented with a 5 year history of progressive proptosis, and initial diplopia. On examination, the best corrected visual acuity was 20/30 on the right eye, 20/20 on the left. A large, non-tender, mobile mass was found at the superomedial region of the right orbit (Fig. 1). The mass was cystic in nature, transilluminable but nonpulsatile. No eggshell cracking sign was evident. The right eye globe was displaced downwards and laterally by the superonasally located mass. Ocular mobility of the right eye was decreased in all directions of gaze, especially on upgaze. The pupillary reactions, colour vision, visual fields and neurological examination were all within normal limits. Slit-lamp and fundal examination showed normal anterior and posterior segments.

Computed tomography (CT) showed a relatively homogeneous mass measuring $34 \times 25 \times 22 \mathrm{~mm}$ at the anterosuperior aspect of the right orbit. It did not enhance on contrast injection. The globe was downwardly and outwardly displaced. A defect was noted in the frontal sinus wall and the mucosa of the sinus adjacent to the mass was thickened. The most likely diagnosis was a mucocoele originating from the right frontal sinus.

Magnetic resonance imaging (MRI) showed a significant degree of mucosal thickening in the right frontal sinus, and it appeared that there was direct 


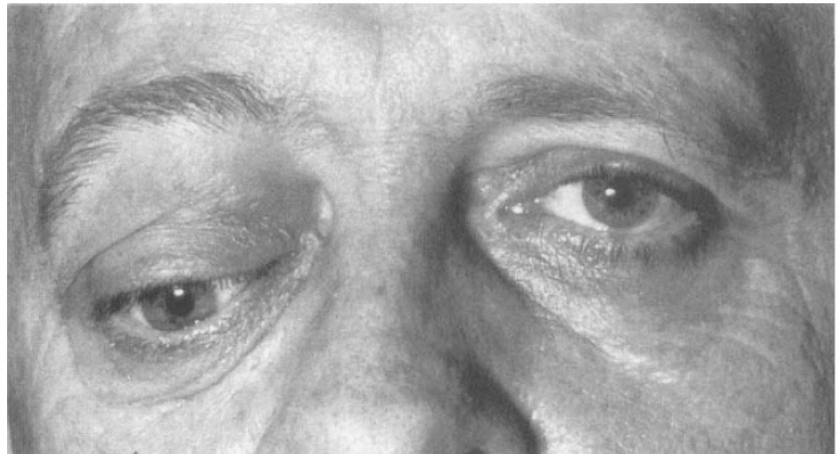

Fig. 1. Pre-operative photograph.

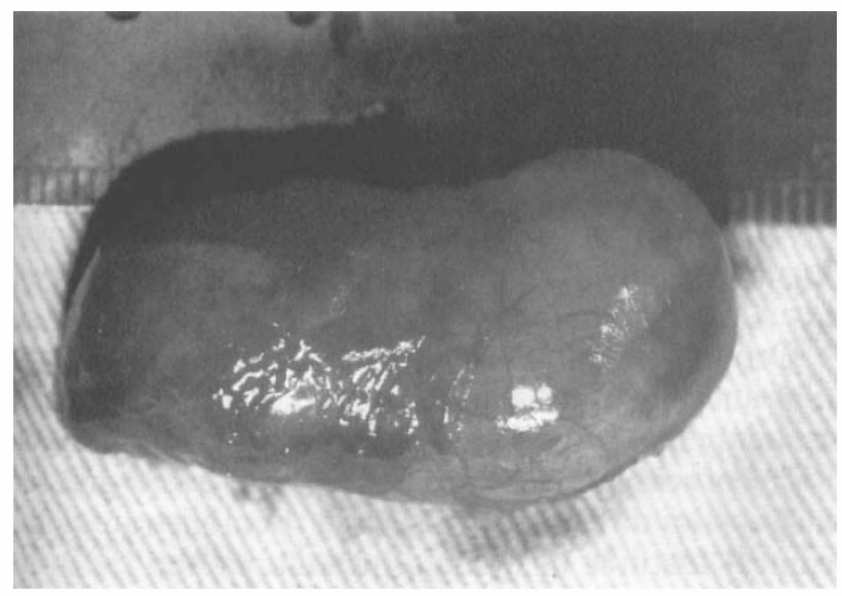

Fig. 3. Gross specimen of the lesion.

communication with the mass (Fig. 2). The left frontal sinus was clear. The lesion had a well-defined margin and appeared to be surrounded by a very thin hypointense capsule.

The orbital mass was removed through a sub-brow incision. The anterior portion of the mass was well exposed when the orbital septum was opened. An oval and well-encapsulated cystic mass was easily dissected and excised en-bloc (Fig. 3). No defect was detected over the orbital roof on palpation. The eye globe was intact and resumed its normal position after the mass had been removed.

Post-operatively the patient had an uneventful recovery, with no diplopia or proptosis. The visual acuity was 20/20 in each eye and binocular vision was restored.

\section{PATHOLOGICAL EVALUATION}

The gross specimen consisted of a cystic mass measuring $3 \mathrm{~cm}$ in the greatest diameter. The surface was smooth with numerous congested vascular structures. On sectioning, the cyst contained strawcoloured fluid. The cyst wall was approximately $1 \mathrm{~mm}$ thick. Most of the inner cyst lining was smooth, but there was a focal solid area of thickening. This plaque-like thickened area was greyish-white in

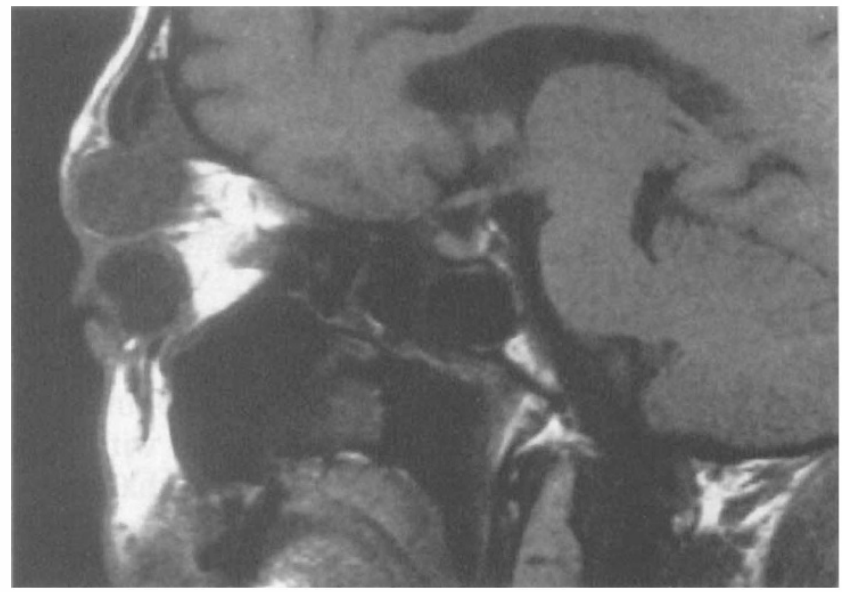

Fig. 2. Sagittal T2-weighted MR image through the right globe and frontal sinus shows a well-circumscribed homogeneous mass pushing the right globe inferiorly. The mass appears to be continuous with the right frontal sinus.

colour and measured $1 \times 0.8 \times 0.3 \mathrm{~cm}$. This structure was attached to the inner lining of the cyst wall. No significant necrosis was seen in the tumour.

Microscopically, the sections showed a cystic wall with no epithelial lining cells. The wall was moderately cellular and composed of proliferating spindle cells and round to ovoid cells (Fig. 4). The spindle cells exhibited neural-type nuclei with scanty inconspicuous eosinophilic cytoplasm, and were arranged in loose vacuolated fascicles (Antoni type B) (Fig. 4, inset $b$ ) and a focal palisading pattern with Verocay bodies (Antoni type A) (Fig. 4, inset a). Mitotic figures were not seen. The vessel wall was slightly thickened with mild hyalinisation. A nerve trunk was noted attached to the thickened area. There was very little collagen present.

A panel of immunocytochemical stains was performed that showed a negative reaction to high and

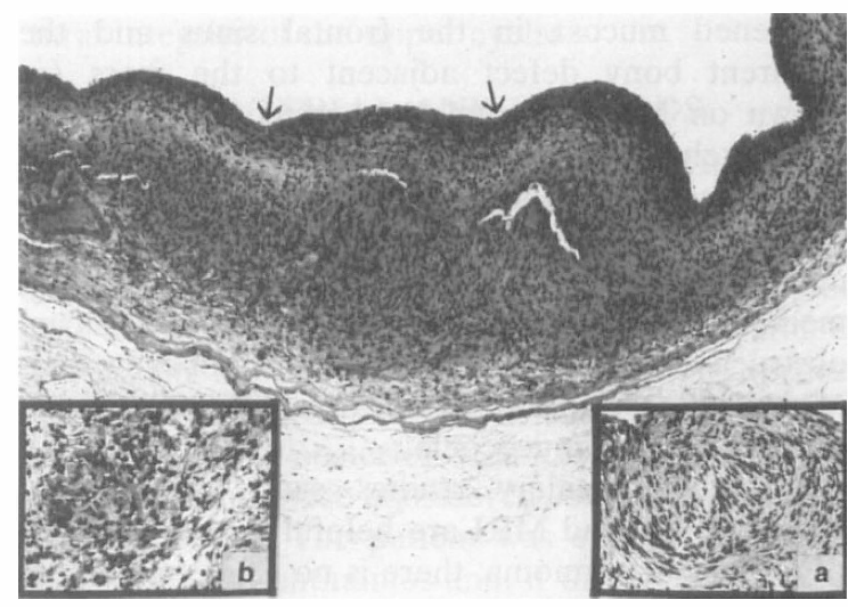

Fig. 4. Section shows a cyst wall with no lining cells on the luminal side (arrows) $(\times 40)$. Spindle-shaped cells are arranged in a palisading Antoni type $A$ pattern (inset a, $\times 100)$ and loose reticular tissue in an Antoni type B pattern (inset $b, \times 100$ ). 
low molecular weight cytokeratin, NSE and desmin but were highly reactive to S-100 antibody and weakly reactive to vimentin. These investigations indicated the lesion originated from Schwann cells of the peripheral nervous system. A schwannoma arising from the nerve trunk with cystic degeneration was diagnosed.

\section{DISCUSSION}

Schwannoma of the orbit is relatively uncommon. It is even rarer to find one of such a large size with total cystic degeneration. There is no single diagnostic clinical feature or specific investigation for orbital schwannoma. Given the unusual degenerative changes and special presentation, the diagnosis of cystic orbital schwannoma can only be made on a histological basis.

The diagnostic difficulty in our case stemmed from the fluid-filled nature of the lesion. The differential diagnoses of cystic orbital tumour include dermoid, mucocoele, haematic cyst, lymphangioma, liposarcoma, fibrous histiocytoma and haemangiopericytoma. The long and relatively asymptomatic clinical course made the diagnosis of malignant tumour very unlikely. For a comparable-sized haemangiopericytoma and fibrous histiocytoma, there would be more evidence of lid and conjunctival congestion as well as optic nerve and extraocular muscle dysfunction. On the other hand, lymphangiomas with a cystic component usually occur in paediatric patients and demonstrate an infiltrating nature in the radiographic investigation.

With a history of minor trauma in that region, the likely cause of a slow-growing paramedian cystic lesion would be a mucocoele, an inclusion cyst or a haematic cyst. Indeed, the findings of a hypodense mass with no contrast enhancement on CT scanning confirmed its cystic nature. Clinically, transillumination could be demonstrated very easily. With the thickened mucosa in the frontal sinus and the apparent bony defect adjacent to the mass (as shown on MRI), frontal sinus mucocoele was the most likely diagnosis.

Although mucocoele was the most likely of the many possible diagnoses, there were some contradictory clinical features. Mucocoele is usually more medially located and is secondary to significant obstructive sinus diseases. The absence of symptoms of sinus disease and the classic eggshell cracking sign were atypical of mucocoele.

Although ancillary studies such as ultrasonography, ${ }^{9} \mathrm{CT}$ and MRI are helpful in the diagnosis of orbital schwannoma, there is no single pathognomonic diagnostic feature among these investigations. In most cases, the diagnosis is based on the characteristic histopathology on excisional biopsy. The gross appearance of schwannoma is usually rather characteristic: a smooth, elongated encapsulated mass. The cut surface is relatively homogeneous, tan or greyish-white with irregular yellow areas and cyst-like spaces. Haemorrhagic areas and thick-walled vessels may also be found. Microscopically, two distinctive cellular arrangements are seen within most schwannomas, which are designated as Antoni A and Antoni B patterns. Verocay bodies and abundant extracellular collagen are generally present. Cystic areas are usually secondary to coalescence of mucinous or microcystic areas in Antoni B tissue of schwannoma. The exact mechanism of cystic degeneration of this type of tumour is not well established and there is no report in the literature documenting such change. The speculative mechanisms include changes in vascular supply resulting in necrosis and cystic change, haemorrhage into tumour with blood resorption causing cyst formation, and hyaline degeneration leading to cyst formation.

Orbital schwannoma, without doubt, is a rare tumour. Its variable presentation and location can certainly make its diagnosis difficult. The pure cystic form of orbital schwannoma poses even greater diagnostic difficulty. This case demonstrates the clinical features of cystic schwannoma and helps to alert the clinician to include cystic schwannoma as a pre-operative differential diagnosis of a slow-growing cystic orbital or periorbital mass.

Key words: Orbit, Cystic, Schwannoma, Proptosis, Mucocoele.

\section{REFERENCES}

1. Rootman J, Goldberg C, Robertson W. Primary orbital schwannomas. Br J Ophthalmol 1982;66:194-204.

2. Mohan H, Sen DK. Orbital neurilemmoma presenting as retrobulbar neuritis. Br J Ophthalmol 1970;54:206-7.

3. Mortada A. Orbital neurilemmoma with café-au-lait pigmentation of the skin. $\mathrm{Br} \mathrm{J}$ Ophthalmol 1968;52: 262-4.

4. Schatz H. Benign orbital neurilemmoma: sarcomatous transformation in von Recklinghausen's disease. Arch Ophthalmol 1971;86:268-73.

5. Ladouceur D, Bergeron D. Cystic schwannoma of the brainstem. Can J Neurol Sci 1989;16:357-60.

6. Wallace CJ, Fong TC, Auer RN. Cystic intracranial schwannoma. Can Assoc Radiol J 1993;44:453-9.

7. Faucett DC, Dutton JJ, Bullard DE. Gasserian ganglion schwannoma with orbital extension. Ophthalmic Plast Reconstr Surg 1989;5:235-8.

8. Andreu J, Alegret $\mathrm{X}$, Perez C, Llauger J. Cystic schwannoma mimicking adrenal tumour. Comput Med Imaging Graph 1988;12:183-5.

9. Barry M, Byrne BA, van Heuven WA. Echographic characteristics of benign orbital schwannomas (neurilemmomas). Am J Ophthalmol 1988;106:194-9. 